\title{
Shunted Piezoelectric Vibration Damping Analysis Including Centrifugal Loading Effects
}

\author{
James B. Min, "Kirsten P. Duffy, Andrew J. Provenza \\ NASA Glenn Research Center \\ *University of Toledo \\ Cleveland, Ohio 44135
}

\begin{abstract}
Excessive vibration of turbomachinery blades causes high cycle fatigue problems which require damping treatments to mitigate vibration levels. One method is the use of piezoelectric materials as passive or active dampers. Based on the technical challenges and requirements learned from previous turbomachinery rotor blades research, an effort has been made to investigate the effectiveness of a shunted piezoelectric for the turbomaninery rotor blades vibration control, specifically for a condition with centrifugal rotation. While ample research has been performed on the use of a piezoelectric material with electric circuits to attempt to control the structural vibration damping, very little study has been done regarding rotational effects. The present study attempts to fill this void. Specifically, the objectives of this study are: (a) to create and analyze finite element models for harmonic forced response vibration analysis coupled with shunted piezoelectric circuits for engine blade operational conditions, (b) to validate the experimental test approaches with numerical results and vice versa, and (c) to establish a numerical modeling capability for vibration control using shunted piezoelectric circuits under rotation. Study has focused on a resonant damping control using shunted piezoelectric patches on plate specimens. Tests and analyses were performed for both non-spinning and spinning conditions. The finite element (FE) shunted piezoelectric circuit damping simulations were performed using the ANSYS Multiphysics code for the resistive and inductive circuit piezoelectric simulations of both conditions. The FE results showed a good correlation with experimental test results. Tests and analyses of shunted piezoelectric damping control, demonstrating with plate specimens, show a great potential to reduce blade vibrations under centrifugal loading.
\end{abstract}

\section{INTRODUCTION}

The requirements for advanced aircraft engine components lead to designs which are more lightweight and efficient, yet more susceptible to excessive vibration, complex dynamic behavior, and uncertain durability and reliability. Structural vibrations also lead to thicker blade designs, increased fuel burn, increased noise, fatigue failures, reduced engine life, reduced safety, and increased maintenance costs. Turbomachinery rotating blades such as fan and compressor blades are subject to high cycle fatigue $(\mathrm{HCF})$ failures as a result of high vibratory stresses. $\mathrm{HCF}$ accounts for fifty-six percent of major aircraft engine failures and ultimately limits the service life of most critical rotating components. An estimated $\$ 400 \mathrm{M}$ is expended annually for HCF related inspection and maintenance of military aircraft alone [1]. Excessive vibration of turbomachinery blades requires damping treatments to mitigate excessive vibration levels which cause HCF problems. Designing damping treatments for rotating blades in an extreme engine environment is a difficult task with various factors such as very high temperatures and centrifugal accelerations. Several damping methods have been investigated by NASA researchers at Glenn Research Center (GRC) for use in aircraft engine blades, including viscoelastic damping [2], impact damping [3, 4], plasma sprayed damping coatings [5], and high-damping high-temperature shape memory alloy materials [6]. Piezoelectric dampers have also been explored as a solution for damping treatment [7-9]. Smart piezoelectric damping technology could alter the dynamic behavior of a structure intelligently to achieve reduced hazardous vibration and associated dynamic stresses, increased life, and enhanced damage tolerance. Additionally, an optimized integrated application of this technology may provide weight and volume savings over conventional damping technology systems. Piezoelectric materials exhibit electromechanical coupling. When a piezoelectric material experiences a strain, a portion of that energy becomes dielectric energy and is stored in the material. In this sense, piezoelectric materials behave electrically as a capacitor. A manufactured material with a high coupling factor is lead zirconate titanate (PZT), and is used in many piezoelectric patches as actuators and dampers [10]. Many researchers have investigated piezoelectric materials in parallel with electric circuits that absorb the vibration energy of components. A survey of smart structures state-of-the-art can be found in a review [11]. A method to determine the effective damping of a shunted piezoelectric material was detailed [12]. Lesieutre [10] described the different types of shunt circuits and how they affect behavior.

For application of piezoelectric dampers to turbomachinery blades, previous work was done at ambient temperatures for non-rotational applications. Hilbert et al. [13] acquired a patent for shunted piezoelectric damping of blades, where piezoelectric patches were placed below the blade platform. Livet [14] examined negative capacitance shunted piezoelectric materials on beams for turbomachinery blade application. Cross and Fleeter [15] tested shunted piezoelectric damping on stator vanes. Piezoelectric networks have also been studied for reduction of mistuned blade vibration levels through blade coupling [16]. Researchers at NASA GRC also carried out the design and implementation of an experimental system that utilized stator vane mounted piezoelectric actuators to control fan-stator interaction noise in a simulated turbo fan engine [17]. These efforts 
have been non-spinning application tests only, and typically used a powered shunt circuit utilizing synthetic inductors or negative capacitance.

Based on the technical challenges and requirements learned from previous turbomachinery rotor blade research, an attempt has been made at GRC to investigate the effectiveness of a shunted piezoelectric material for the turbomaninery rotor blades application, specifically for a condition with centrifugal rotation. The study has focused on a resonant damping control using shunted piezoelectric patches on plate specimens. Tests and analyses were performed for both non-spinning and spinning loading. A finite element (FE) modeling effort has also been made using the ANSYS Multiphysics [18] code which can model piezoelectric coupled-field elements and electric circuit elements for the resistive and inductive circuits.

Some earlier test and analysis work conducted at GRC on the piezoelectric beam specimens is described [7]. Passive and active resonant damping control results showed that shunted piezoelectric damping control techniques have a great potential to reduce plate vibrations under centrifugal loading at room temperature. The FE results also showed a good correlation with experimental test results. The validated numerical models and the experimental test results obtained from the study allow us to extend our current effort for design and optimization of more complex turbomachinery rotor blade systems.

The target application for the present study is a titanium-alloy cold-side compressor or fan blade, which operates at temperatures up to about $600^{\circ} \mathrm{F}$. Resonant frequencies are on the order of 100 to $10,000 \mathrm{~Hz}$. The blade structure loss factor, $\eta$, is typically on the order of $10^{-3}$ or less for titanium alloy blades, depending on the vibration mode, but $10^{-2}$ or more would be desirable for resonant stress reduction. This can be accomplished by incorporating a high damping material within or on the surface of the blade. If the damping material is placed on the blade surface, it needs to be very thin so that it does not adversely affect the aerodynamics. Fundamental modes (e.g. first bending or first torsion) are more easily damped with traditional methods such as platform dampers. While there is interest in developing new damping techniques for higher-order modes where resonant stresses occur near the blade tips, the focus of the current effort was on the third bending mode.

Centrifugal loading also causes blade stiffening, leading to changes in resonant frequency for some modes with rotational speed. The piezoelectric shunt circuit design will need to provide damping over the desired speed range [9]. A configuration of the current study was chosen to demonstrate the effectiveness of shunted piezoelectric materials for damping centrifugally-loaded plates.

While ample research has been performed on the use of a shunted piezoelectric material connected to electric circuits for controlling vibration damping in a stationary frame, very little study has been done to assess rotational effects in that subject. The present study attempts to fill this void by demonstrating the feasibility of piezoelectric damping on rotating plates through design, build, and testing of piezoelectric blade or blade-like test specimens in a laboratory environment. Specifically, the objectives of this study are: (a) to create and analyze finite element models for harmonic forced response vibration analysis coupled with shunted piezoelectric circuits under rotation, (b) to validate the experimental test approaches by a comparison with numerical results or vice versa, and (c) to obtain a numerical modeling capability for vibration control using shunted piezoelectric circuits under rotation.

\section{MATERIALS AND APPROACHES}

In this study, cantilevered plates with a piezoelectric patch connected with passive shunt circuits shown in Figure 1 were tested and analyzed to measure vibration reduction versus a baseline undamped plate. Tests were conducted on a vibration exciter (shaker) at room temperature. The plate specimen of Ti-6Al-4V, 8" long x 0.75 " wide x 0.078 " thick, clamped at one end, was analyzed and tested. To simulate a fan blade type structure, the test article configuration of a simple rectangular plate was fabricated.

Figure 2 shows an actual photograph of shunt circuit connected piezoelectric plate specimen on the vibration exciter. The piezoelectric patch was placed at a location optimized for the third bending mode (3B) which is the target mode in this study.

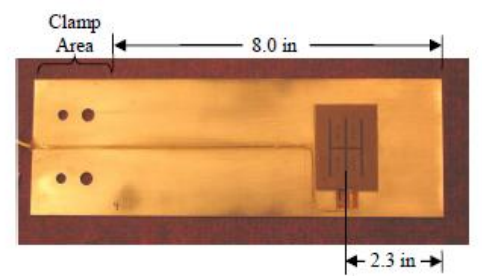

Figure 1: Piezoelectric patched specimen with geometric dimensions

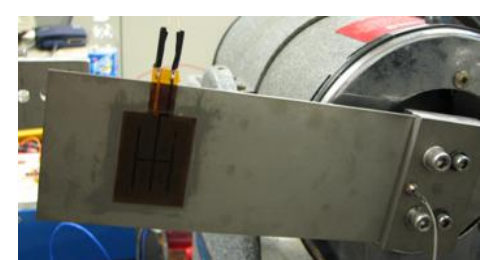

Figure 2: Actual photograph of piezoelectric plate specimen on the shaker

For passive vibration damping, the piezoelectric elements are connected to a specific electrical network consisting of a dissipative shunt circuit [12]. One method is the tuned shunt where a circuit made with an inductor (L) and a resistor (R) in series is connected to the capacitance of the piezoelectric elements. Optimal damping is obtained by tuning the electrical resonance to the frequency of the chosen structural mode with $\mathrm{R}$ and $\mathrm{L}$. 


\subsection{Shunted piezoelectric passive vibration control experiments}

Non-spinning, room-temperature vibration tests were performed and analyzed for two types of circuits: resistive circuits (R circuit) and inductive circuits (RL circuit). The inductor can be a simple coiled wire, which is fully passive. Both circuit types were tested on a vibration exciter (shaker), and the resistive circuit was also tested under centrifugal loading. Table 1 lists the shunt circuit configurations used for analysis and testing.

Table 1: Shunt Circuit Configurations [9]

\begin{tabular}{|c|c|c|c|}
\hline Test & $\begin{array}{c}\text { Shunt Circuit } \\
\text { Configuration }\end{array}$ & $\begin{array}{c}\text { Shunt } \\
\text { Resistance }\end{array}$ & $\begin{array}{c}\text { Shunt } \\
\text { Inductance }\end{array}$ \\
\hline \multirow{5}{*}{ Shaker } & 3B-O-S & Open circuit & -- \\
& 3B-S-S & Short circuit & -- \\
& $3 B-R-S$ & $0-22 \mathrm{k} \Omega$ & -- \\
\hline \multirow{2}{*}{ Spin Rig } & $3 B-R L-S$ & $0-22 \mathrm{k} \Omega$ & $0.38 \mathrm{H}, 0.69 \mathrm{H}, 1.27 \mathrm{H}$ \\
\hline
\end{tabular}

The circuit components were chosen to maximize damping based on the Hagood and von Flotow loss factor equations [12], and based on shaker results. One plate was analyzed and tested on a shaker with the piezoelectric patch in the open circuit condition, short circuit condition, with a resistive shunt circuit, and with an inductive shunt circuit. A pair of plates was then tested in the NASA-GRC Dynamic Spin Facility (spin rig) in the open circuit condition and with the optimal resistor.

The inductors were open-core wound inductors manufactured in-house. The inductor for a 0.69 Henry $(\mathrm{H})$ inductance is shown in Figure 3, which is quite large since the target frequency is only $700-800 \mathrm{~Hz}$. This was chosen because $3^{\text {rd }}$ bending frequency below the excitation bandwidth of $1000 \mathrm{~Hz}$ [9].

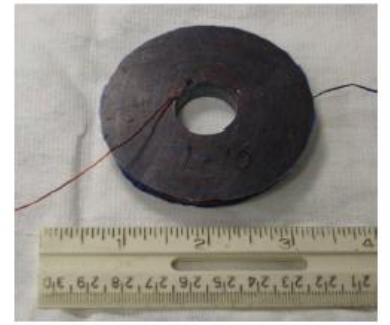

Figure 3: Photograph of in-house inductor of $0.69 \mathrm{H}$

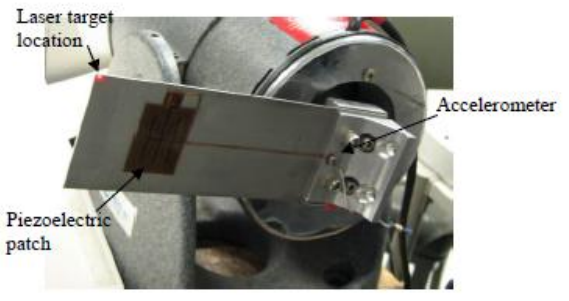

Figure 4: Bench test setup

A cantilevered test plate was clamped to a shaker as shown in Figure 4. An accelerometer was placed on the clamp to measure the excitation level. A laser vibrometer was used to measure the velocity of the plate tip. An analyzer was used in the swept-sine mode to generate a signal to the shaker. The analyzer measured the signals to the shaker, as well as the accelerometer and laser vibrometer signals. The damping was calculated from the transfer function of the tip velocity to the clamp acceleration, as well as from the tip velocity frequency response.

Spin testing was performed in the Dynamic Spin Facility at NASA GRC. Figure 5 shows a photograph of the spin facility, and Figure 6 shows a pair of plates attached to a hub on the vertical rotor. Testing was done in a 0.01 psia vacuum at room temperature. The rotor was fully levitated on three conventional active magnetic bearings. An axial magnetic bearing supports the weight of the rotor system. The two other magnetic bearings support the rotor radially, and also provide a radial excitation to the shaft, transmitting vibration to the plates. At a typical excitation level, the base of the plate gets approximately a one-g excitation with a bandwidth of about $1000 \mathrm{~Hz}$. A non-contacting stress measurement system (NSMS), with laser displacement probes, measures the plate tip deflections while the rotor spins.

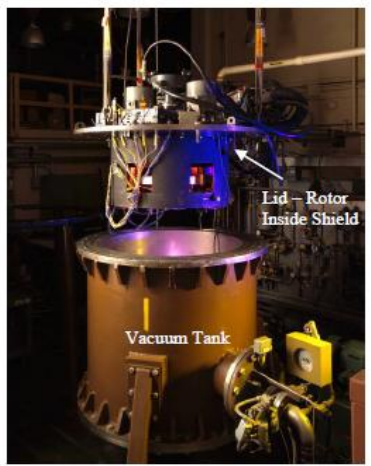

Figure 5: Photograph of spin facility

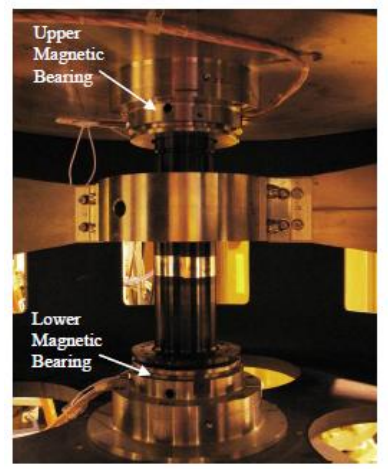

Figure 6: A pair of plates attached to rotor hub

For this test, the magnetic bearing control system provided an engine order excitation frequency - the excitation frequency was an integer order of the rotation speed. Two identical plate configurations were tested at the same time, and were 
oriented directly opposite each other on the rotor. The shunt resistors were placed on the blade hub, directly inboard of the plate clamps. Testing was performed at speeds of zero rpm to $4000 \mathrm{rpm}$.

\subsection{Finite element models (FEM) for shunted piezoelectric harmonic vibration damping}

FEM-circuit coupled piezoelectric simulation models based on electric charge balance were developed with the ANSYS Multiphysics code. The objectives of the modeling effort were: (a) to predict the modal frequencies and mode shapes of experimental test specimens under rotation, (b) to conduct harmonic forced vibration response analyses on the plate models coupled with shunted piezoelectric circuits for blade rotational conditions, (c) to validate the experimental test approaches employed with a comparison of numerical and experimental test results vice versa, and (d) to develop a numerical modeling capability for vibration control using shunted piezoelectric circuits.

A cantilever plate with a piezoelectric patch as shown in Figure 1 was modeled with passive shunt circuits to measure vibration reduction versus a baseline undamped plate. The poling direction of the piezoelectric patch was in the direction of thickness. The titanium plate was meshed with SOLID95 which has twenty nodes with three degrees of freedom per node: translations in the nodal $\mathrm{x}, \mathrm{y}$, and $\mathrm{z}$ directions. The piezoelectric patch was meshed with SOLID226 which has twenty nodes with four degrees of freedom including the piezoelectric effect per node: translations in the nodal $\mathrm{x}$, $\mathrm{y}$, and $\mathrm{z}$ directions, and the electrical potential $V$. In addition, CIRCU94 elements were used to model the resistor and inductor connected to each electrode of the piezoelectric patch.

Conversion of material properties of piezoelectric materials for use in ANSYS Multiphysics was challenging because of differences between manufacturer-supplied data and the format required by ANSYS. This section provides a frame work of our FE modeling techniques for shunt circuit-fed piezoelectric vibration damping analyses under rotation. It outlines the prestressed modal analysis equations, piezoelectric finite element equations, general constitutive equations of piezoelectric materials, piezoelectric circuit element equations, and piezoelectric harmonic response analysis equations. It also covers converting manufacturer's data to ANSYS format data for the stiffness matrix, dielectric constants, and piezoelectric constants.

As the piezoelectric patch is polarized in its thickness direction but operates in the plane dimension, its input material properties are orthotropic and consist of stiffness, dielectric and piezoelectric matrices. The piezoelectric effect results in elongation and compression of the two surfaces (electrodes) of the piezoelectric plate, proportional to the electrical voltage and pulsates at the excitation frequency. When this piezoelectric patch is bonded to the plate, it transmits a pulsed bending moment into the plate, which then vibrates at the same frequency. When this frequency coincides with one of the plate natural frequencies, resonance occurs. A perfect adhesive bonding between actuator and plate was assumed in the model. Frequency response functions (FRF) were generated from a full harmonic analysis.

\subsubsection{Natural frequency with nonlinear rotational effects of spin softening and stress stiffening}

To accurately calculate the resonant frequencies for spinning rotor blade, the analysis was carried out with a prestressed modal analysis method prior to conducting the harmonic piezoelectric-circuit analysis. Also, a spin softening model was used to examine whether the plate could survive at given rotational speeds. Experimental testing was performed at speeds from zero rpm to $4000 \mathrm{rpm}$ even though the facility has the capability of spinning at up to $20,000 \mathrm{rpm}$.

In the model, rotational velocities are combined with the element mass matrices to form a body force load vector. As a small deflection analysis cannot directly account for change in geometry, the effect for large deflection was accounted for by an adjustment of the stiffness matrix. Thus, the large deflection effects are included in a small deflection solution. This allowed adjusting the stiffness of a rotating piezoelectric plate to account for dynamic mass effects. Equilibrium of the system and centrifugal forces on the mass using small deflection logic can be expressed as

$$
[K]\{U\}=\left\{\Omega^{2}\right\}[M]\{r\}
$$

where:

$$
\begin{aligned}
& \{U\}=\text { radial displacement of the mass from the rest position } \\
& \{r\}=\text { radial rest position of the mass with respect to the axis of rotation } \\
& \Omega=\text { angular velocity of rotation } \\
& {[K]=\text { stiffness matrix }} \\
& {[M]=\text { mass matrix }}
\end{aligned}
$$

However, to account for large deflection effects, Eq. (1) must be expanded to:

$$
[K]\{U\}=\left\{\Omega^{2}\right\}[M]\{r+U\}
$$

Rearranging terms,

$$
\left([K]-\left\{\Omega^{2}\right\}[M]\right)\{U\}=\left\{\Omega^{2}\right\}[M]\{r\}
$$

Defining: 


$$
\begin{aligned}
& {[\bar{K}]=[K]-\left\{\Omega^{2}\right\}[M]} \\
& {[\bar{F}]=\left\{\Omega^{2}\right\}[M]\{r\}}
\end{aligned}
$$

Eq. (1) becomes simply,

$$
[\bar{K}]\{U\}=[\bar{F}]
$$

$[\bar{K}]$ is the stiffness needed in a small deflection solution to account for large deflection effect, and $[\bar{F}]$ is the same as that derived from small deflection logic. Thus, the large deflection effects are included in a small deflection solution. This decrease in the effective stiffness matrix is called spin-softening [19].

Additionally, when the strains in a material exceed more than a few percent and the rotation rates are large, the changing geometry due to this deformation can no longer be neglected. Analyses which include this effect are called large strain-large rotation analyses for stress stiffening contribution to the updated stiffnesses. Stress stiffening (also called geometric stiffening) is the stiffening (or weakening) of a structure due to its stress state. This stiffening effect normally is to be considered for thin structures with bending stiffness very small compared to axial stiffness. This stress stiffness matrix was added to the regular stiffness matrix in order to give total stiffness for accuracy.

The governing equation of motion for rotation including inertia forces and moments can be expressed in matrix form as:

$$
[M]\{\ddot{U}\}+[C]\{\dot{U}\}+([\bar{K}]+[\bar{S}])\{\mathrm{U}\}=[\bar{F}]
$$

where:

$$
\begin{aligned}
& {[M]=\text { global mass matrix }=\sum_{e=1}^{e=n} M^{e}} \\
& e=\text { element } 1,2, \ldots, n \\
& {\left[M^{e}\right]=\text { element mass matrix }=\int_{V}[N]^{T}[N] \rho d V} \\
& {[N]=\text { shape function matrix }} \\
& \rho=\text { element density } \\
& {[C]=\text { global damping matrix }=\sum_{e=1}^{e=n} C_{e}} \\
& n=\text { number of elements }
\end{aligned}
$$

$[\bar{K}]=$ global stiffness of piezoelectric plate including spin softening stiffness due to centrifugal force

$[\bar{S}]=$ stress stiffening contribution matrix due to large strain and large rotation $[\bar{S}]=\int_{V}[G]^{T}[\tau][G] d V$

$[G]=$ matrix shape function derivatives

$[\tau]=$ matrix of current Cauchy (true) stresses in global coordinate

$[\bar{F}]=$ global load vector due to rotating body force, vibration shaker excitation force, and piezoelectric voltage and charge

The equation of motion for an undamped system, expressed in matrix notation is:

$$
[M]\{\ddot{U}\}+[\tilde{K}]\{U\}=\{0\} \quad \text { where }[\tilde{K}]=[\bar{K}]+[\bar{S}]
$$

Note that $[\tilde{K}]$ includes prestress effects due to plate spinning and stress stiffening. Eq. (7) was used for natural frequency and mode shape determination. For a linear system, free vibration is harmonic of the form:

where:

$$
\{U\}=\left\{\varphi_{i} \operatorname{cost} \omega_{i} t\right.
$$

$\left\{\varphi_{i}\right\}_{i}=$ eigenvector representing the mode shape of the $i^{\text {th }}$ natural frequency

$\omega_{i}=i^{\text {th }}$ natural frequency (cycles per second)

$$
t=\text { time }
$$

Thus, Eq. (7) becomes:

$$
\left(-\omega_{i}^{2}[M]+[\tilde{K}]\right)\{\varphi\}_{i}=\{0\}
$$


This equality gives the solution:

$$
\left|[\tilde{K}]-\omega_{i}{ }^{2}[M]\right|=0
$$

The Block Lanzos Method was used to solve this eigenvalue problem. This method uses a combination of the automated shift strategy and the Sturm Sequence Check strategy. The two strategies aim to reduce the number of iterations in solving the eigenvalue problem yet maintain good accuracy. After obtaining the frequencies and mode shapes of prestressed piezoelectric plates under rotational conditions, the next step was to build the coupled piezoelectric-circuit harmonic vibration analysis model to obtain the piezoelectric-circuited plate forced vibration responses under rotation.

\subsubsection{Piezoelectric finite element method}

A finite element method for piezoelectric medium is briefly described. Piezoelectric FEM equations can be written in terms of nodal displacement $\{U\}$ and nodal electric potential $\{\phi\}$ for each node. The displacement and potential for each element can be expressed, respectively, as

$$
\begin{aligned}
\{U\} & =\left[N_{u}\right]^{T}\left\{U_{e}\right\} \\
\{\phi\} & =\left[N_{\phi}\right]^{T}\left\{\phi_{e}\right\}
\end{aligned}
$$

where

$$
\begin{aligned}
& {\left[N_{u}\right]^{T}=\text { displacement shape function (transposed) }} \\
& {\left[N_{\phi}\right]^{T}=\text { electrical potential shape function (transposed) }} \\
& \left\{U_{e}\right\}=\text { nodal displacement } \\
& \left\{\phi_{e}\right\}=\text { nodal electric potential }
\end{aligned}
$$

The mechanical strains, $\{S\}$, are related to the nodal displacements through the derivative of the shape function $\left[B_{u}\right]$

$$
\{S\}=\left[B_{u}\right]\{U\}
$$

where $\left[B_{u}\right]$ is the strain-displacement matrix. Similarly the electrical field $\{E\}$ is related to the nodal potential as

$$
\{E\}=-\left[B_{\phi}\right]\{\phi\}
$$

where $\left[B_{\phi}\right]$ is the electric field-displacement matrix.

The equations of motion for a piezoelectric body can be derived from the principle of minimum potential energy by means of a variational functional. The resultant equations can be represented in matrix form from the assembly of all the individual finite element equations. The equations are written in terms of displacement $\{U\}$ and electrical potential $\{\phi\}$ at the nodal points. Forcing functions are expressed in terms of structural loads $\{F\}$ and electrical loads $\{Q\}$, resulting in the equilibrium equations below [20-22]. The equation of motion corresponding to the piezoelectric actuator and the plate structure can be assembled in a global system coordinate, which includes the degrees of freedom of the piezoelectric actuator (voltages and displacements) and the degrees of freedom of the plate structure (displacements) as follows:

$$
\begin{aligned}
& {\left[\boldsymbol{M}_{u u}\right]\{\ddot{U}\}+\left[\boldsymbol{C}_{u u}\right]\{\dot{U}\}+\left[\boldsymbol{K}_{u u}\right]\{U\}+\left[\boldsymbol{K}_{u \phi}\right]\{\phi\}=\{F\}} \\
& {\left[\boldsymbol{K}_{u \phi}\right]^{T}\{U\}+\left[\boldsymbol{K}_{\phi \phi}\right]\{\phi\}=\{Q\}}
\end{aligned}
$$

This same expression can also be expressed in the matrix form:

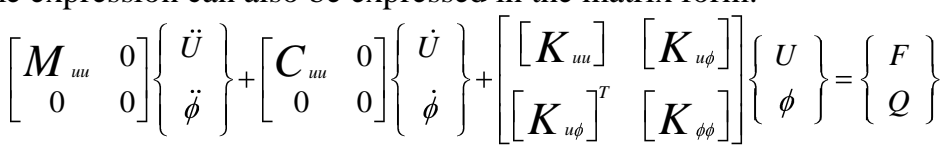

where

$$
\begin{aligned}
& {\left[\boldsymbol{K}_{u u}\right]=\iiint \int_{\Omega_{e}}\left[\boldsymbol{B}_{u}\right]^{T}[c]\left[\boldsymbol{B}_{u}\right] d V} \\
& {\left[\boldsymbol{K}_{u \phi}\right]=\iiint_{\Omega_{e}}\left[\boldsymbol{B}_{u}\right]^{T}[e]\left[\boldsymbol{B}_{\phi}\right] d V}
\end{aligned}
$$




$$
\begin{aligned}
& {\left[K_{\phi \phi}\right]=\iiint_{\Omega_{e}}\left[B_{\phi}\right]^{T}[\varepsilon]\left[B_{\phi}\right] d V} \\
& {\left[M_{u u}\right]=\rho \iiint_{\Omega_{e}}\left[N_{u}\right]^{T}\left[N_{u}\right] d V} \\
& {\left[C_{u u}\right]=\beta\left[K_{u u}\right]}
\end{aligned}
$$

where

$\Omega_{e}$-finite element domain

$\rho-$ piezoelectric density

$\left[K_{u u}\right]$ - mechanical stiffness matrix

$\left[B_{u}\right],\left[B_{\phi}\right]$ - derivatives of FEM shape functions

$\left[K_{u \phi}\right]$ - piezoelectric coupling matrix

[c]-elastic coefficients

$\left[K_{\phi \phi}\right]$ - dielectric stiffness matrix

[e]-piezoelectric coefficients

$\left[M_{u u}\right]$ - mass matrix

$[\varepsilon]$ - dielectric coefficients

$\left[C_{u w}\right]$ - mechanical damping matrix

$\beta$-damping coefficient

\subsubsection{Constitutive relationship of piezoelectric materials}

The basic constitutive relationship of piezoelectric materials is outlined below. A piezoelectric model requires permittivity (or dielectric constants), the piezoelectric matrix, and the elastic coefficient matrix to be specified as material properties. The constitutive relationship usually given by manufacturers or published reports is in the following form:

$$
\begin{aligned}
& \{S\}=\left[S^{E}\right]\{T\}+[d]\{E\} \\
& \{D\}=[d]^{t}\{T\}+\left[\varepsilon^{T}\right]\{E\}
\end{aligned}
$$

ANSYS requires data in the following form:

$$
\begin{aligned}
& \{T\}=\left[C^{E}\right]\{S\}-[e]\{E\} \\
& \{D\}=[e]^{t}\{S\}+\left[\varepsilon^{S}\right]\{E\}
\end{aligned}
$$

where

$$
\begin{aligned}
& \{T\}=\text { stress vector (six components } x, y, z, x y, y z, x z) \\
& \{S\}=\text { strain vector (six components } x, y, z, x y, y z, x z) \\
& \{D\}=\text { electric displacement vector } \text { (three components } x, y, z) \\
& \{E\}=\text { electric field vector (three components } x, y, z \text { ) } \\
& {\left[c^{E}\right]=\text { stiffness matrix evaluated at constant electric field, i.e. short circuit }} \\
& {[e]=\text { piezoelectric matrix relating stress/electric field }} \\
& {[e]^{t}=\text { piezoelectric matrix relating stress/electric field (transposed) }} \\
& {\left[\varepsilon^{s}\right]=\text { dielectric matrix evaluated at constant strains, i.e. mechanically clamped }} \\
& {[d]=\text { piezoelectric matrix relating strain/electric field }} \\
& {[d]^{t}=\text { piezoelectric matrix relating strain/electric field (transposed) }} \\
& {\left[\varepsilon^{T}\right]=\text { dielectric matrix evaluated at constant stress, i.e. mechanically free }}
\end{aligned}
$$

In order to convert the manufacturer's data presented in the form of Eqs. (16) \& (17) to ANSYS notation (Eqs. (18) \& (19)), Eq. (16) needs to be based on stress rather than strain. Eq. (16) can be rearranged as:

$$
\{T\}=\left[S^{E}\right]^{-1}\{S\}-\left[S^{E}\right]^{-1}[d]\{E\}
$$

Since Eq. (17) relates electric displacement to strain rather than stress, Eq. (20) can then be plugged back into Eq. (17) to yield:

$$
\{D\}=[d]^{t}\left[S^{E}\right]^{-1}\{S\}+\left[\varepsilon^{T}\right]-[d]^{t}\left[S^{E}\right]^{-1}[d]\{E\}
$$

Upon comparison of Eqs. (20) \& (21) with Eqs. (18) \& (19), one can obtain the relationship between manufacturer-supplied data and ANSYS-required values: 


$$
\begin{aligned}
& {\left[C^{E}\right]=\left[S^{E}\right]^{-1}} \\
& {\left[\mathcal{E}^{S}\right]=\left[\mathcal{E}^{T}\right]^{-[d]^{t}\left[S^{E}\right]^{-1}[d]}} \\
& {[e]=\left[S^{E}\right]^{-1}[d]}
\end{aligned}
$$

These equations form the basis of the conversion routines for converting manufacturer's data to ANSYS data for stiffness matrices, dielectric constants, and piezoelectric constants. Note that the manufacturer's data has mechanical vectors in the form $\{x, y, z, y z, x z, x y\}$ whereas ANSYS's mechanical vectors are in the form $\{x, y, z, x y, y z, x z\}$.

\subsubsection{Permittivity matrix (Dielectric constants)}

Dielectric constants represent the diagonal components $\varepsilon_{11}, \varepsilon_{22}$, and $\varepsilon_{33}$ respectively of the permittivity matrix $\left[\varepsilon^{S}\right]$.

(The superscript "s" indicates that the constants are evaluated at constant strain.) The permittivity matrix evaluated at constant strain is input into ANSYS. Typically, manufacturers' data has permittivity evaluated at constant stress, so conversion is necessary.

After evaluating Eq. (23), the permittivity matrix has only diagonal terms:

$$
\left[\varepsilon^{s}\right]=\left[\begin{array}{rrr}
\mathcal{E}_{11}^{s} & 0 & 0 \\
& \mathcal{E}_{11}^{s} & 0 \\
& & \boldsymbol{\varepsilon}_{33}^{s}
\end{array}\right]=\boldsymbol{\varepsilon}_{o}\left[\begin{array}{rrr}
\boldsymbol{K}_{11}^{s} & 0 & 0 \\
& \boldsymbol{K}_{11}^{s} & 0 \\
& & \boldsymbol{K}_{33}^{s}
\end{array}\right]
$$

where $K_{11}^{S}=\frac{\varepsilon_{11}^{S}}{\varepsilon_{0}}$ is relative permittivity.

Although there is a choice of inputting permittivity as an absolute value $\varepsilon_{33}^{T}$ or relative value $K_{33}^{T}$, the relative value is recommended. Relative values were used in the present work.

\subsubsection{Piezoelectric matrix [e]}

Usually, manufacturers' data gives $[d]$, which relates mechanical strain to electric field. However, ANSYS requires $[e]$, relating mechanical stress to electric field, so conversion is required. Note from Eq. (24), a relationship between $[e]$ and $[d]$ is established as follows where assuming polarization in the 3-axis (z-direction) and symmetry in the unpolarized directions ( $d_{32}=d_{31}$ and $\left.d_{24}=d_{15}\right)$.

$$
[d]^{t}=\left[\begin{array}{cccccc}
0 & 0 & 0 & 0 & 0 & d_{15} \\
0 & 0 & 0 & 0 & d_{15} & 0 \\
d_{31} & d_{31} & d_{33} & 0 & 0 & 0
\end{array}\right]
$$

This matrix can be used with $\left[S^{E}\right]^{-1}=\left[C^{E}\right]$ to evaluate $[e]$ which will become,

$$
[e]^{t}=\left[\begin{array}{cccccc}
0 & 0 & 0 & 0 & 0 & \boldsymbol{e}_{15} \\
0 & 0 & 0 & 0 & \boldsymbol{e}_{15} & 0 \\
\boldsymbol{e}_{31} & \boldsymbol{e}_{31} & \boldsymbol{e}_{33} & 0 & 0 & 0
\end{array}\right]
$$

This matrix relates the electric field to stress.

\subsubsection{Stiffness Matrix [C]}

The stiffness matrix is a symmetric matrix that specifies the stiffness coefficients:

$$
\left.{ }_{[C}\right]^{E}=\left[\begin{array}{lllllll}
c_{11} & & & & & \\
c_{21} & c_{22} & & & & \\
c_{31} & c_{32} & c_{33} & & & \\
c_{61} & c_{62} & c_{63} & c_{66} & & \\
c_{41} & c_{42} & c_{43} & c_{46} & c_{44} & \\
c_{51} & c_{52} & c_{53} & c_{56} & c_{54} & c_{55}
\end{array}\right]
$$

Appropriate numerical values of the matrix coefficients were input for modeling the plate specimens illustrated in Section 2.2.5. 


\subsubsection{Piezoelectric circuit elements}

The piezoelectric circuit element in ANSYS, CIRCU94, simulates basic linear electric circuit components that can be directly connected to the piezoelectric finite element domain. It is suitable for the simulation of circuit-fed piezoelectric dampers for vibration control under the influence of harmonic (sinusoidally varying) forces, currents, displacements, and voltages. CIRCU94 elements were used to model resistor (R) and inductor (L) elements connected to each electrode of the piezoelectric patch. Any number of combinations of loadings is permitted and they need not be in phase, but they must be at the same frequency. The finite element equations for the resistor, inductor, capacitor and current source were derived using the nodal analysis method $[23,24]$. To be compatible with the system of piezoelectric finite element equations, the nodal analysis method was adapted to maintain the charge balance at each node:

$$
[K]\{V\}=\{Q\}
$$

where

$$
\begin{aligned}
& {[K]=\text { stiffness }(\text { capacitance) matrix }} \\
& \{V\}=\text { vector of nodal voltages }(\text { to be determined) } \\
& \{Q\}=\text { load vector of nodal charges }
\end{aligned}
$$

In a harmonic analysis, the stiffness matrix becomes

$$
C\left[\begin{array}{cc}
1 & -1 \\
-1 & 1
\end{array}\right]\left\{\begin{array}{l}
V_{1} \\
V_{2}
\end{array}\right\}=\left\{\begin{array}{l}
0 \\
0
\end{array}\right\}
$$

for capacitor,

$$
j \omega\left(-\frac{1}{\omega^{2} R}\right)\left(\begin{array}{cc}
1 & -1 \\
-1 & 1
\end{array}\right)\left\{\begin{array}{l}
V_{1} \\
V_{2}
\end{array}\right\}=\left\{\begin{array}{l}
0 \\
0
\end{array}\right\}
$$

for a resistor,

$$
\left(-\frac{1}{\omega^{2} L}\right)\left(\begin{array}{cc}
1 & -1 \\
-1 & 1
\end{array}\right)\left\{\begin{array}{l}
V_{1} \\
V_{2}
\end{array}\right\}=\left\{\begin{array}{l}
0 \\
0
\end{array}\right\}
$$

and for a inductor

where

$$
\begin{aligned}
& j=\text { imaginary unit, } j^{2}=-1 \\
& \omega=\text { driving frequency } \\
& V_{1} \text { and } V_{2} \text { are the shunt voltage as degrees of freedom at the two nodes for each } R \text { and L elements }
\end{aligned}
$$

Equations 34-36 can be readily added to Eq. (15) for harmonic analysis [23].

\subsubsection{Piezoelectric harmonic response analysis under harmonic excitation}

The harmonic response analysis solves time-dependent equations of motion shown in Eq. (15) for structures undergoing steady-state vibration. All points in the structure are moving at the same known frequency, however, not necessarily in phase. It is known that the presence of damping causes phase shifts. Therefore, the displacements $\{U(t)\}$ and electrical potential $\{\phi(t)\}$ may be defined as:

where

$$
\{U(t)\}=\{U\} e^{-j \omega t+\psi} \quad\{\phi(t)\}=\{\phi\} e^{-j \omega t+\psi}
$$

$$
\begin{aligned}
& \omega=\text { driving frequency } \\
& \psi=\text { phase shift } \\
& t=\text { time }
\end{aligned}
$$

Force and charge are expressed as

$$
\begin{aligned}
& \{F(t)\}=\{F\} e^{-j \omega t+\psi} \\
& \{Q(t)\}=\{Q\} e^{-j \omega t+\psi}
\end{aligned}
$$


Note that the $\{Q(t)\}$ is related to the current $I$ by

$$
\{Q(t)\}=(1 / j \omega)(I) e^{-j \omega t+\psi}
$$

Substituting Eqs. (33)-(35) in the piezoelectric constitutive Eq. (15) yields

$$
\left[\begin{array}{cc}
K_{U U}+j \omega C_{U U}-\omega^{2} M_{U U} & K_{U \phi} \\
K_{U \phi}^{T} & K_{\phi \phi}
\end{array}\right]\left\{\begin{array}{l}
\hat{U} \\
\hat{\phi}
\end{array}\right\}=\left\{\begin{array}{l}
\hat{F} \\
\frac{1}{j \omega} I
\end{array}\right\}
$$

where the superscript " $\wedge$ " represents the complex matrices.

Note that peak harmonic response occurs at forcing frequencies that match the natural frequencies of the piezoelectric plates.

\subsubsection{Geometric data of piezoelectric plate specimens}

A titanium plate test specimen with a rectangular cross-section was studied. The dimensions and the physical properties are given in Table 2. A piezoelectric patch was bonded onto the upper face of the plate. The piezoelectric properties of PZT-5 [25] are given in Table 3. The poling direction for the piezoelectric patch is in the direction of thickness, and the mechanical boundary condition of the structure is clamped. On each interface between piezoelectric and the plate, the electrical potential is forced to zero. Since the target mode in this study was the third bending mode, a piezoelectric patch was placed at a location of high strain energy for this mode to provide optimal effectiveness of the patch for the structural damping. A single Midé PZT-5A qp10w15 piezoelectric patch was bonded to one side of the plate. The patch was centered at 2.3 inches from the tip, in a high modal strain area for the third bending (3B) mode. The patch was 1.5 inches long, 2.0 inches wide, and 0.015 inches thick. The piezoelectric material itself within the patch has dimensions of 1.31 inches long, 1.81 inches wide, and 0.010 inches thick. The geometric sketches of the model are shown in Figure 7.

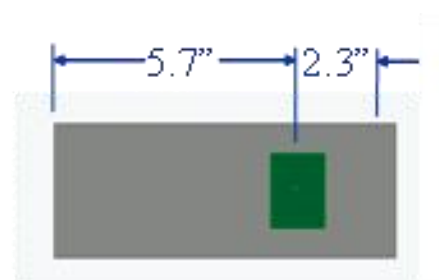

(a)

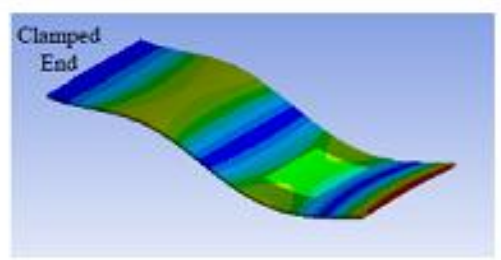

(b)

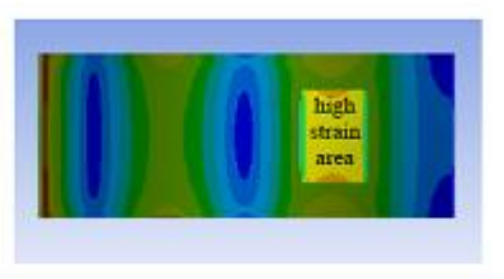

(c)

Figure 7: (a) Geometric sketch of active part of rectangular piezoelectric plate configuration for FEM, (b) Plot of modal deformation, and (c) Equivalent modal strain of 3B mode at zero rotation speed

Table 2: Ti 6Al-4V plate characteristics

\begin{tabular}{|l|l|}
\hline Length & 8 inch \\
\hline Width & 3.2 inch \\
\hline Thickness & 0.077 inch \\
\hline Young's modulus & $1.52 \mathrm{E}+07 \mathrm{psi}$ \\
\hline Poisson's ratio & 0.3 \\
\hline Density & $0.16 \mathrm{lb} / \mathrm{in}^{3}$ \\
\hline
\end{tabular}

\begin{tabular}{|c|c|c|c|c|c|c|c|c|c|c|c|c|c|c|c|c|c|c|c|c|c|c|}
\hline & & & & & & & & & & & & & & & & & & & & & & \\
\hline & 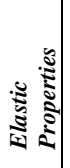 & 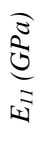 & 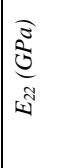 & 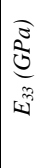 & 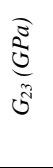 & $\frac{\bar{s}}{0}$ & 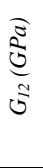 & $\beth$ & $=$ & $\stackrel{n}{=}$ & 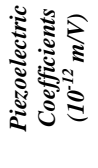 & $\widehat{x}$ & $\widetilde{8}$ & 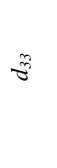 & తే & $\frac{2}{2}$ & 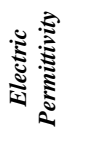 & $\stackrel{\stackrel{心}{\omega}}{\stackrel{\omega}{\omega}}$ & 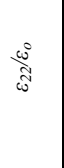 & 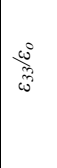 & 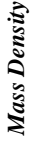 & 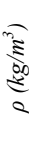 \\
\hline : & & $\approx$ & 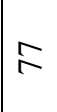 & 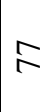 & $\begin{array}{l}0 \\
\stackrel{\lambda}{2}\end{array}$ & ڤ̊. & 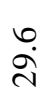 & $\stackrel{n}{0}$ & $\stackrel{n}{0}$ & $\stackrel{m}{0}$ & & 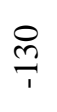 & 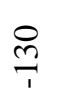 & ల్లి & $\stackrel{\hat{n}}{n}$ & & & 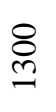 & 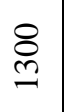 & 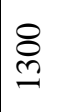 & & $\underset{\gtrless}{\stackrel{2}{\gtrless}}$ \\
\hline
\end{tabular}

Table 3: Piezoelectric properties $\left(\varepsilon_{o}=8.85 \times 10^{-12} \mathrm{farad} / \mathrm{m}\right.$, electric permittivity of air) [25] 


\section{RESULTS AND DISCUSSIONS}

Piezoelectric finite element analyses and experimental tests were performed to determine the relative effectiveness of fully passive shunt circuit damping techniques on rotating piezoelectric plate specimens. The test specimen configuration consists of a piezoelectric patch attached to a vibrating plate at a location of high stress. An electrical circuit was attached to the patch, which can dissipate the electrical energy.

\subsection{Experimental Test Results}

This section describes the experimental test results obtained from non-spinning bench tests and spin rig tests, respectively.

\subsubsection{Non-Spinning Bench Test Results}

Figure 8 shows some shaker test data on the same rectangular plate. The velocity of the plate tip was measured. The third bending loss factor, $\eta$ was 0.00156 (i.e. critical damping ratio $\zeta=7.8 \times 10^{-4}$ ) with an open circuit (no circuit connected to the patch).
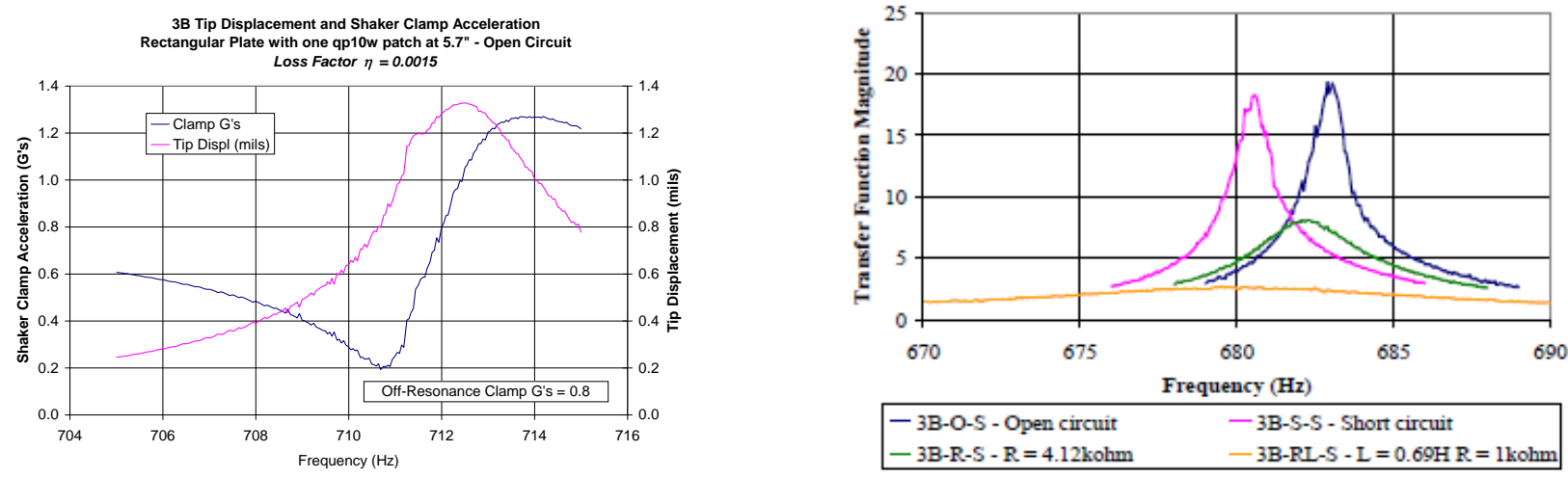

Figure 8: Shaker data on rectangular plate

Figure 9: Transfer functions - plate tip velocity to clamp acceleration $(\mathrm{in} / \mathrm{s} / \mathrm{g})$

A plate was tested on the shaker as shown in Figure 2. The bench testing of the rectangular plates was performed to determine the optimal inductor size for the third bending resonance mode. As predicted, there was optimal damping at a resistance of approximately $4 \mathrm{k} \Omega$. A resistance of $4.12 \mathrm{k} \Omega$ was used in spin test. Vibration levels were reduced for the 3B resonance levels by as much as 90 percent with a properly tuned inductive shunt circuit. Figure 9 shows the resonant peak reduction with shunt circuits. With the $0.69 \mathrm{H}$ inductance and a resistance of $2 \mathrm{k} \Omega$ or less, the desired damping can be achieved in a non-spinning test.

\subsubsection{Spin Rig Test Results}

A rectangular titanium plate finite element model spinning up to $4000 \mathrm{rpm}$ was modeled to see the stress state at the plate hub against the materials strength. The maximum von Mises equivalent stress value at $4000 \mathrm{rpm}$ was approximately 14 ksi at the corners of the plate hub area as shown in Figure 10. An acceptable margin of the safety was calculated for spinning up to $4000 \mathrm{rpm}$.

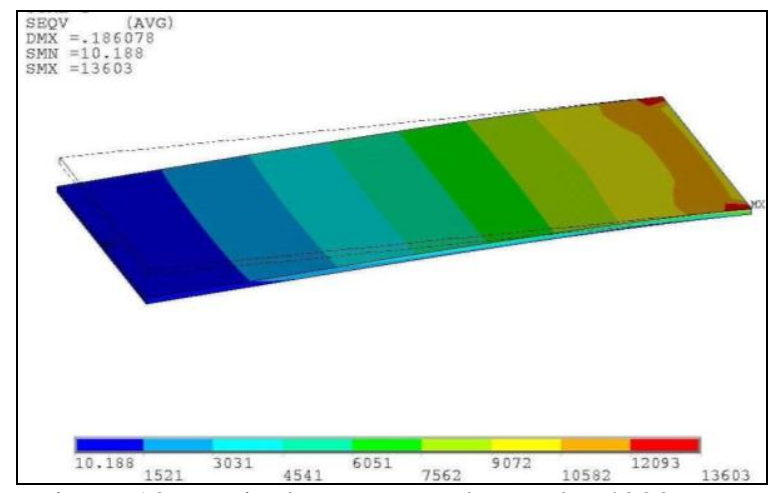

Figure 10: Equivalent stress value under $4000 \mathrm{rpm}$

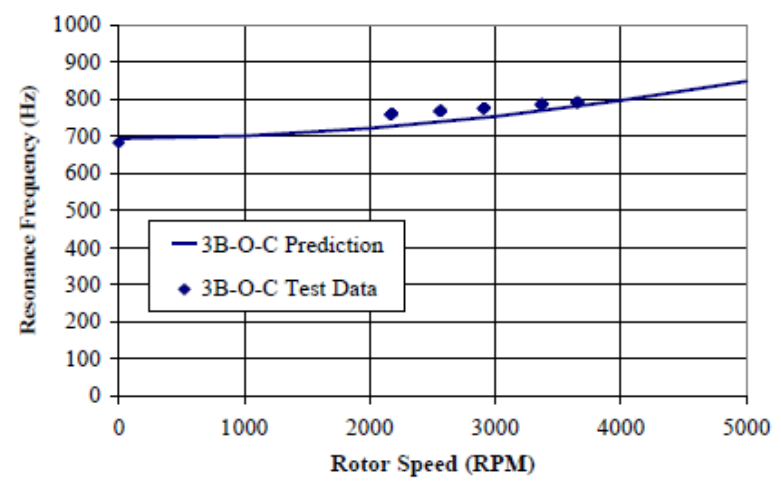

Figure 11: Comparison of third bending (3B) resonance frequencies with FEM predictions and test data

Before performing spinning vibration tests, the plates were run to a maximum speed of $4000 \mathrm{rpm}$ for one minute in the Dynamic Spin Facility. They were then removed from the rig and inspected to check the structural integrity of the plates, piezoelectric material, wiring, and bonding materials. In addition, the piezoelectric capacitance was checked before and after 
vibration spin testing. This test proved that spin testing could be performed using these flat plate test articles. Figure 11 shows both the predicted and experimental resonance frequencies as a function of rotor speed for the 3B-O-C (3B open circuit) configuration. Each experimental data point shown represents a resonance occurring when the engine order excitation frequency matched the third bending mode resonance frequency. The correlation between modeling and experiment is good. The frequencies also increase with rotor speeds.

Spin tests up to $3800 \mathrm{rpm}$ were performed on both open circuit and resistive shunt circuit plates. Spin tests at 3800 $\mathrm{rpm}$ using a resistive shunt $(R=4120 \Omega)$ reduced vibrations by approximately 20 percent versus the open circuit mode. Figure 12 shows the damping increase due to the resistive shunt circuit. An adequate 3B response with the inductive shunt circuit piezoelectric plate specimens did not appear to be attainable using the current spin rig test setup. More spin tests will be required to demonstrate the resistive and inductive circuit effectiveness under centrifugal load.

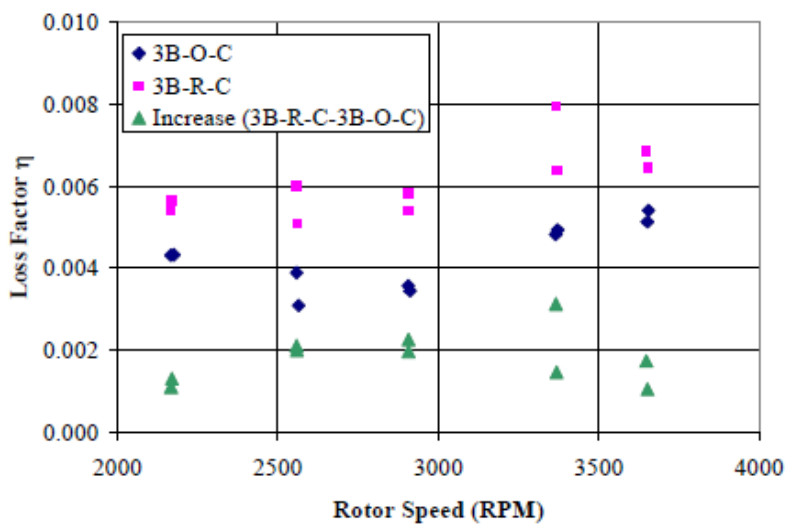

Figure 12: Spin test data - loss factor (damping) increased using resistive shunt circuit

\subsection{FE Analysis Results and Comparisons to Experimental Test Results}

This section describes the electrical shunt circuit-fed piezoelectric finite element results and their comparisons to those of the experimental tests.

\subsubsection{Non-Spinning Results}

An ANSYS finite element-electrical circuit coupled piezoelectric vibration simulation model was developed using electric circuit components. To perform a coupled piezoelectric-electrical circuit analysis, the piezoelectric circuit element, CIRCU94, was used to model the resistor and inductor.

For modeling the circuit coupled piezoelectric titanium plate structure, the electric circuits were directly connected to the 3-D piezoelectric coupled-field 20-node brick elements, SOLID226, through a set of common nodes or by coupling separate nodes. The titanium plate was meshed with 20-node brick elements, SOLID95. The location of the circuit with respect to the distributed piezoelectric domain is arbitrary and does not affect the analysis results. The intent was to create a resonant circuit by shunting the inherent capacitance (C) of the piezoelectric with a resistor and inductor in series forming a RLC circuit to control the 3B mode of the piezoelectric plate. This patch was fully covered by electrode poled through its thickness and actuated lengthwise so that it was operating in the transverse mode. In addition to the source load, the only other load is a zero voltage specification at the ground nodes.

The experimentally measured shaker excitation shown in Figure 8 was approximated in the piezoelectric finite element analysis with a constant base excitation of $0.6 \mathrm{~g}$ to simulate actual shaker excitation. The tip displacements obtained from the finite element models were compared with the experimental test results. Three numerical results of zero rotational speed cases were compared to those of the experimental test data as shown in Figure 12.

A constant loss factor, $\eta$ of 0.00156 (i.e. critical damping ratio $\zeta=7.8 \times 10^{-4}$ ) experimentally determined with an open circuit for the 3B resonance mode, was used for inherent structural modal damping of the 3B mode. There is a different inherent structural damping ratio for each resonance mode. This pure structural damping value was maintained for other circuit cases studied. The frequency response function (FRF) obtained from the FE model for open circuit case was compared with that of the experimental test. The agreement between finite element model and experimental test was excellent as shown in Figure 12 (top two curves). Based on this inherent structural modal damping agreement for the open circuit case, other circuit cases were numerically analyzed for structural damping plus damping induced by the shunted piezoelectric circuits. Those results are shown in the same Figure 12. Clearly it can be seen that vibration levels were reduced by as much as 90 percent with a properly tuned inductive shunt circuited piezoelectric patch.

The result of an open-circuit case displays excellent agreement between modeling and test. While numerical and experimental test results show a good tendency overall, a larger difference in the results was observed with resistive and inductive circuit cases comparing to the open circuit case. The tendency of overestimated damping values obtained by the FEM may possibly be a result of uncertainty in piezoelectric material properties used in the model. Equally, many sources of the uncertainties involved in the experimental test results such as adhesive bonding, wiring elements, and also inconsistent shaker 
excitations as shown in Figure 8, possibly may be part of this difference as well. Since all the piezoelectric material properties necessary for FE modeling were not available from the piezoelectric materials manufacturer for the experimental tests, some approximations were necessary for inputting the piezoelectric material properties for FE modeling. Hence, differences in experimental test and FE model possibly comes from: (1) discrepancy in piezoelectric properties and clamp boundary conditions; (2) imperfections in bonding; (3) modal damping measurements variation; (4) possible experimental wiring signal effects; (5) inconsistent shaker excitations; (6) and imperfection in the connection of piezoelectric patch and electric circuitry in the experimental tests.

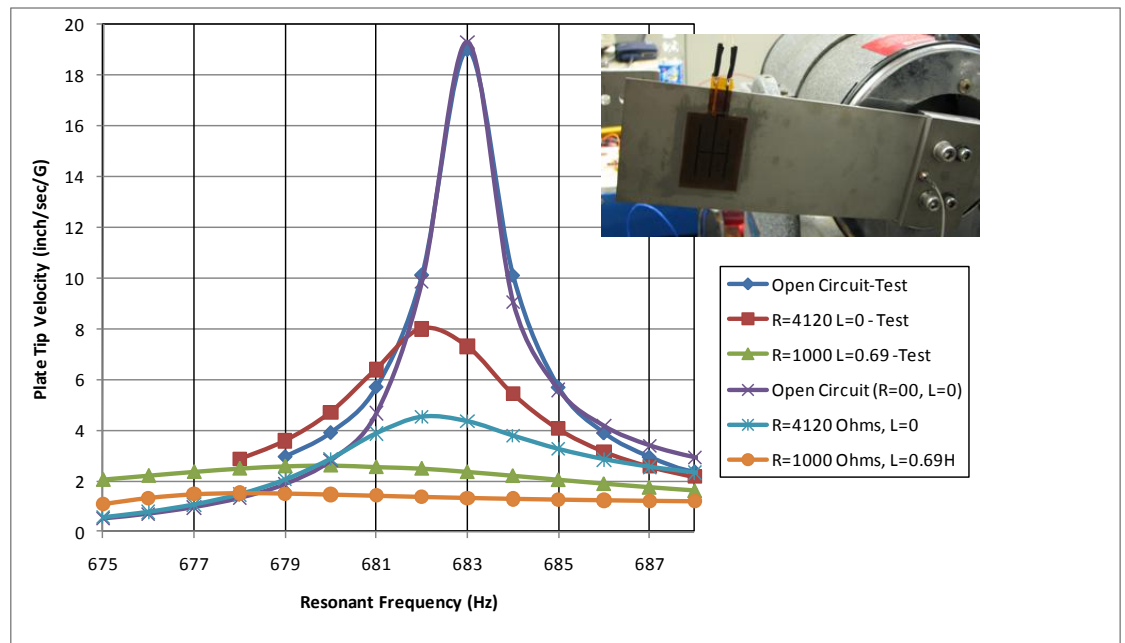

Figure 12: Shaker bench test results: harmonic responses of FEM vs. experimental test (R- resistance $\Omega, L$ - inductance $H$ ); Note: units of abscissa $(\mathrm{Hz})$ for resonant frequency and ordinate $(\mathrm{in} / \mathrm{sec} / \mathrm{g}$ ) for plate tip velocity

\subsubsection{Spinning Results}

In order to predict dynamic characteristics of the rotating coupled piezoelectric-circuit plate in the spin rig, finite element modal analyses were performed. Vibration amplitudes of the rotating piezoelectric plate were examined to prevent excess vibration. The prestressed modal frequencies of open-circuit piezoelectric plate, including spin softening and stress stiffening effects, were obtained numerically as a function of rotational speed. The Campbell diagram in Figure 13 shows a prediction of the rotational speeds at which fundamental plate modes will be excited. Resonance excitation will occur if the excitation is set at a multiple of the rotational speed and the rotor is spinning at a speed where this engine order line crosses the resonance curve. For the speed range shown, only multiples of engine order (E) higher than 10E will excite the 3B mode.

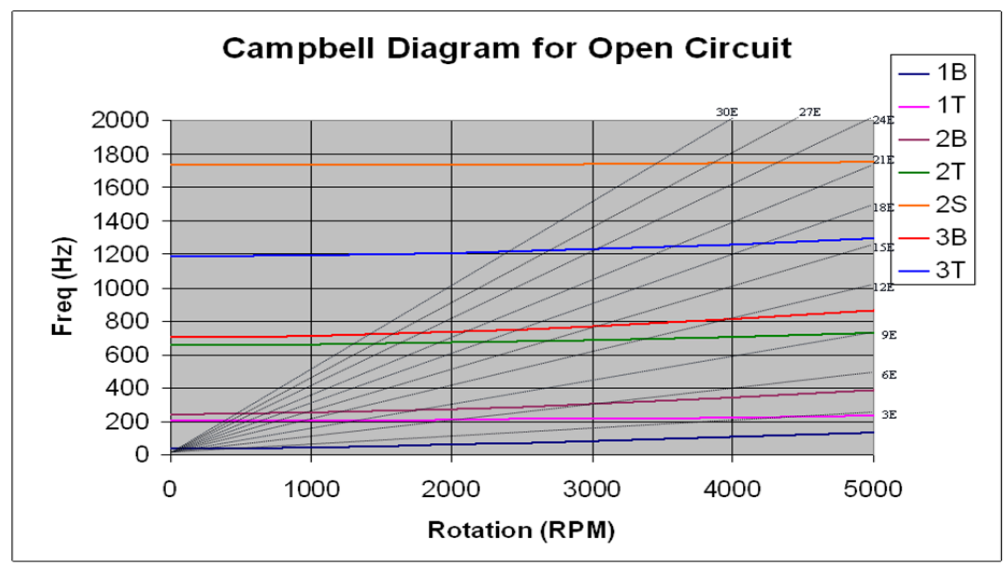

Note: rectangular plate with a patch at 3B modal location

Figure 13: Campbell diagram for open-circuit piezoelectric plate

One of the objectives of the finite element modeling was to predict frequency response for comparison with the frequency response functions (FRF) obtained by experimental spin tests. However, FRFs (transfer functions) from the experimental spin tests were not available since a non-contacting stress measurement system (NSMS) with laser displacement probes was used to measure the plate tip displacement while the rotor spins (Duffy et al, 2009). The transfer functions by the experimental spin tests will be obtained in the future using either a slip ring or a wireless inductive power transfer device being developed at GRC. Hence, Figures 14 and 15 show the results obtained only from the numerical spin test simulations. 
The FE harmonic response analysis was performed for a piezoelectric-circuit plate excited by a harmonic vibration of $0.6 \mathrm{~g}$ acceleration at different rotational speeds. Figure 14 shows the frequency response functions numerically obtained for variable rotational speeds with open circuit case. The open-circuit piezoelectric plate having a 3B patch was spun to $5000 \mathrm{rpm}$. The results show that the plate tip velocities increase with rotational speed. Damping, however, decreases with increasing rotor speed. Centrifugal loading not only affects the resonance frequencies, but also affects the damping levels, typically causing reduced damping with increased rotor speed. Frequency shifts are also observed as the rotational speeds increase. These frequency shifts are mainly attributed to a stiffness change as a result of spin softening and stress stiffening effects.

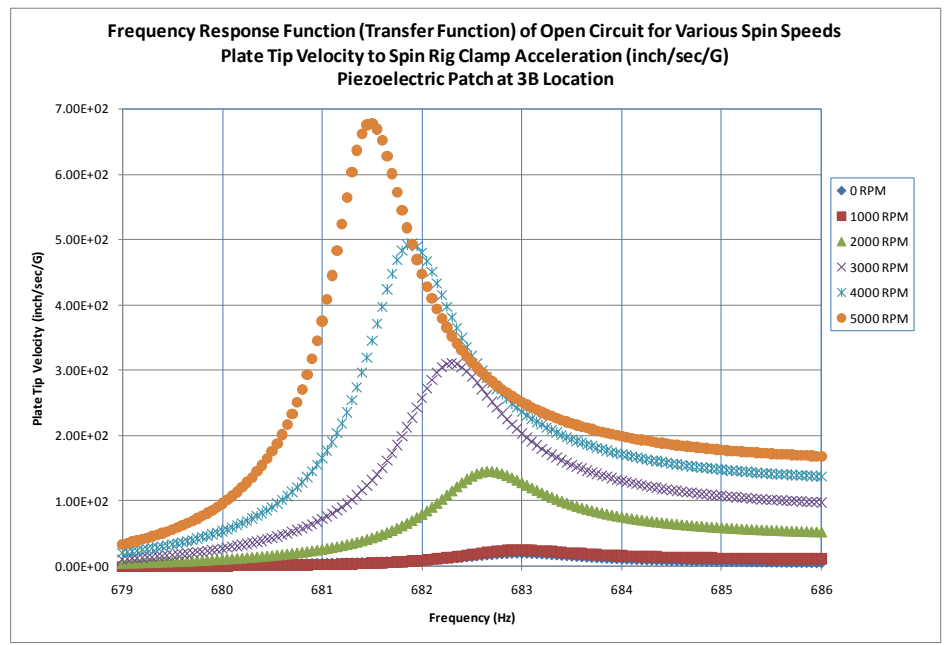

Figure 14: Frequency response function (transfer function) for open circuit vs. rotational speeds; Note: zero rpm response function curve is hidden behind the curve of $1000 \mathrm{rpm}$.

The effectiveness of a shunted resistive circuit versus speed is shown with Figure 15. These results were obtained for a 3B case. The result shows that a resistance equal to $4120 \Omega$ is the most effective for reducing vibration amplitude at approximately $1000 \mathrm{rpm}$. The resistance value can be changed to obtain similar high damping at a higher rotor speed, if desired.

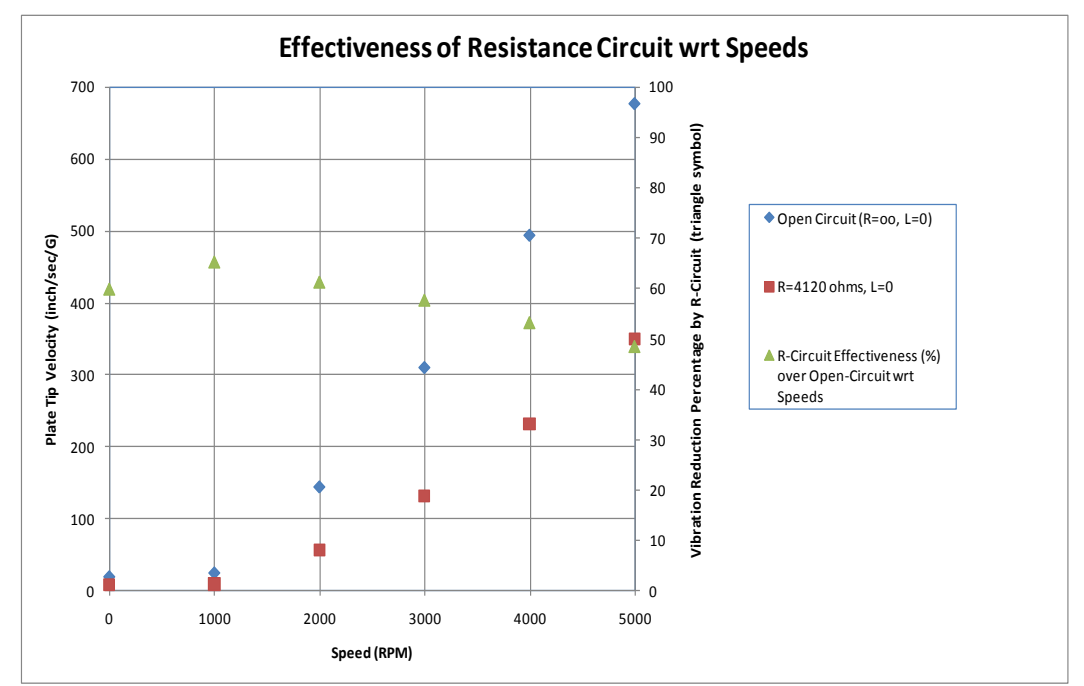

Figure 15: Effectiveness of shunted resistive circuit vs. rotational speeds

FE simulation was also conducted for the inductive circuit piezoelectric plate. A circuit made with an inductor (L) and a resistor $(\mathrm{R})$ in series connected to the capacitance of piezoelectric patch was modeled for the inductive shunt circuited plates under rotation. Experimental spin rig tests with an inductive shunt circuited piezoelectric plate were performed for controlling 3B. However, an adequate response from the current spin rig test setup did not appear to be attainable. Instead, the $2^{\text {nd }}$ bending (2B) response with a 3B patch was attainable from the spin testing using the current spin test setup. Therefore, FE modal and frequency response analyses for the inductive circuit piezoelectric plate were performed to predict the response functions of the $2 \mathrm{~B}$ with a 3B patch under rotations. In spite of that, the FRF (transfer function) of $2 \mathrm{~B}$ from the experimental spin tests was not available for a comparison with those obtained numerically since a NSMS with laser displacement probes can measure the plate tip deflection only while the rotor spins. Figure 16 shows the numerical results of the $2^{\text {nd }}$ bending $(2 \mathrm{~B})$ harmonic 
responses with a resistance value equal to $1500 \Omega$ and an inductance value equal to $4 \mathrm{H}$. A comparison with FE results indicates that vibration reduction percentage is practically independent of rotor speed with an inductive shunt circuit. It shows that the RL inductive shunted circuit can still reduce the vibration as much as 90 percent in contrast to the open circuit. Figures 17 and 18 show the specific transfer functions for zero rotation and $1000 \mathrm{rpm}$ for a comparison against open circuit responses, respectively. Both cases clearly illustrate a significant reduction in the peak amplitude when inductive shunt circuit is used. The inductive shunt circuits will enable the damping to remain high over the operating speed range.

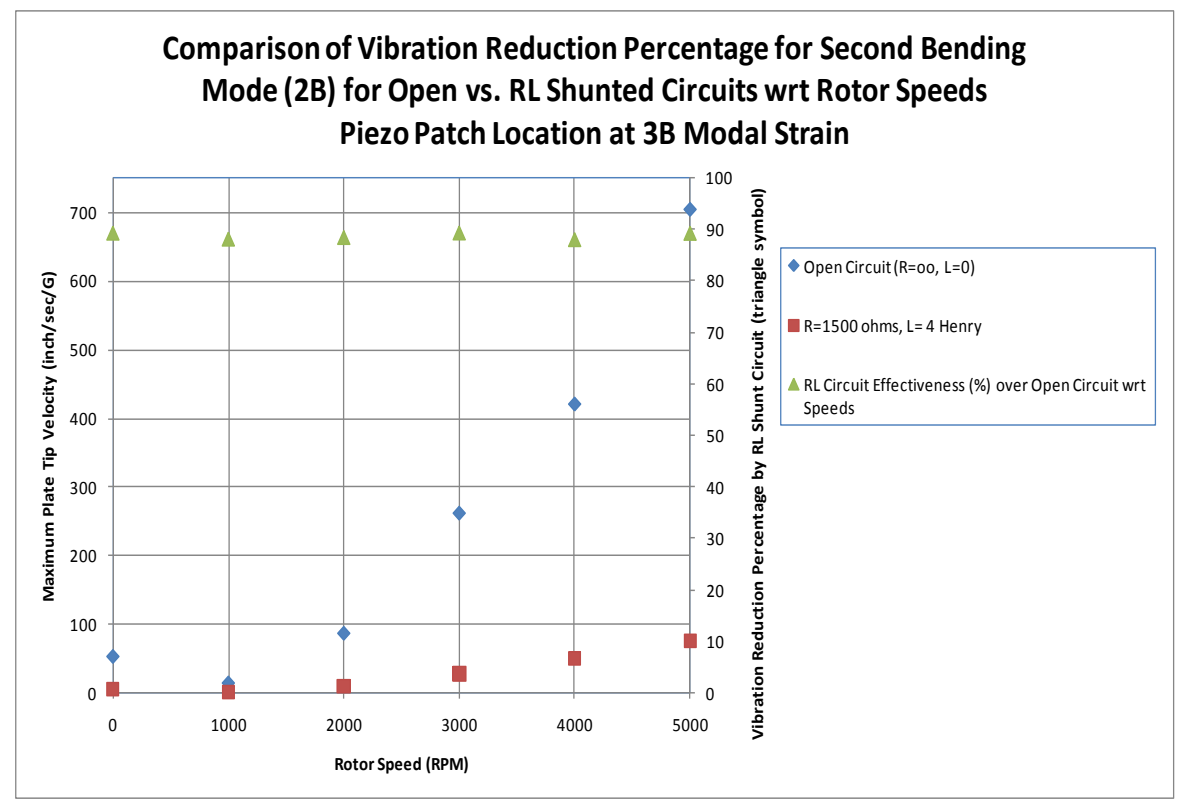

Figure 16: Numerical results of 2B harmonic responses with a patch at 3B modal location

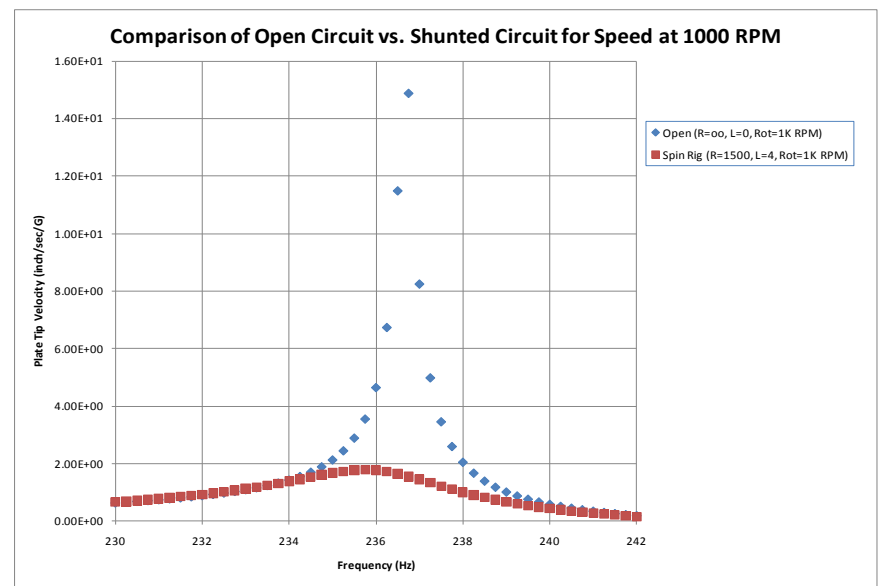

Figure 17: Comparison of 2B transfer function for open circuit vs. shunted inductive circuit with 1000rpm

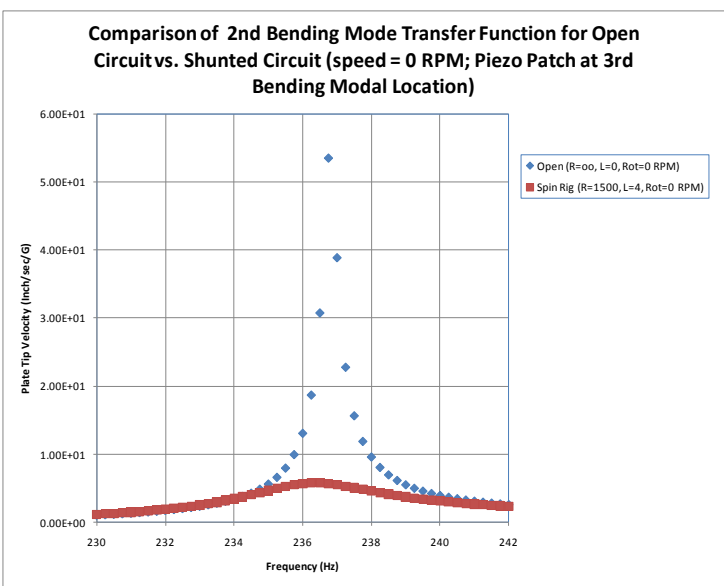

Figure 18: Comparison of 2B transfer function for open circuit vs. shunted circuit with zero rotation

Based on the results shown in the current paper with a rotating piezoelectric plate, a study is extended for the real piezoelectric blade specimens under rotation.

\section{SUMMARY}

A numerical and experimental study using the electrical circuit-fed piezoelectric patched metal plate under rotation was performed to develop and demonstrate a shunted piezoelectric vibration damping technique to reduce vibrations in turbomachinery fan or compressor blades.

Spin and shaker tests were conducted on passively-shunted piezoelectric-damped rectangular plates. Third bending resonance amplitude levels were greatly reduced with a properly tuned inductive and resistive shunt circuit in a non-spinning test.

Finite element harmonic response analysis models were developed and utilized along with the experimental tests to validate the test data or vice versa. The result of an open-circuit case displayed excellent agreement between modeling and 
experimental test. While numerical and experimental test results compare favorably, a larger difference in the results was observed with resistive and inductive circuit cases when compared to an open-circuit case. The tendency of overestimated damping values obtained by the shunt circuit piezoelectric finite element models may possibly be a result of uncertainties in the piezoelectric material properties and approximations of the boundary conditions used in the model. Equally, many other sources of uncertainties involved in the experimental tests that are not included in the analysis, such as imperfections in bonding, modal damping measurements variation, possible experimental wiring signal effects, inconsistent shaker excitations, and also wiring work involved in connection of piezoelectric patch and electric circuitry, possibly contribute to difference as well. Additional work is required to examine effects of uncertainties mentioned.

An effective resistive circuit for the third bending mode vibration reduction was identified for plate specimens under rotation. A resistive shunt circuit reduced vibrations by approximately 20 percent in experimental spin tests and 65 percent in numerical simulations. Again, a tendency of overestimated damping values with the finite element results for a resistive shunt circuit case under rotation may be a result of uncertainty in piezoelectric material properties used in the models and other uncertainties involved in measurements in the experimental tests.

The finite element analysis results indicate that vibration reduction percentages by an inductive shunt circuit do not vary with rotational speed. Nevertheless, it shows that the inductive shunted circuit under rotation can still reduce the vibration as much as 90 percent against the open circuit. Whereas this behavior should be verified when the inductive circuit test data from the spin rig tests in progress are available, a significant reduction in the peak amplitude is predicted by FEM. The inductive shunt circuits will enable the damping to remain high over the operating speed range.

The present numerical and experimental test results also indicate that resistive and inductive shunt piezoelectrics have the ability to reduce plate vibrations significantly under rotation. A resistive shunt alone may not be sufficient to achieve the desired damping goal. The tuned inductive circuit with a wound inductor yielded very high damping in shaker tests and numerical simulations, and will be tested and validated in the spin rig in the near future. The addition of an inductor to form a tuned inductive circuit for rotation should increase damping considerably.

A new numerical tool developed for vibration control using shunted resistive and inductive piezoelectric circuits for plate specimen under rotation allows for the prediction of modal frequencies and mode shapes of the plate specimens under rotation, simulation of the harmonic forced response vibration analysis for blade operational conditions, and validation of experiment results.

Based on the numerical and experimental results shown, electrical circuit-fed finite element piezoelectric modeling techniques and spin rig experiments should facilitate the study of more challenging and realistic tests of piezoelectric damped turbomachinery blades.

\section{FUTURE WORK}

The modeling techniques explained herein will be extended to real blade specimens. These specimens will be spintested and analyzed in a similar fashion. Continuous vibration data will be available as the experimental test setup will include a slip ring. Test blades will be composite and metal, and piezoelectric patches will be surface-mounted and embedded. The use of synthetic inductive shunt circuits may also be explored. These circuits can be smaller in size, but will require power in the rotating frame. The capability to transfer power to the test rotor exists using a wireless inductive power transfer device. Newer state-of-the-art high temperature piezoelectric patches may also be explored.

\section{Acknowledgments}

Support provided by the NASA Fundamental Aeronautics Program, Subsonic Fixed Wing Project is acknowledged. Other members (G. Stefko, M. Bakhle, B.B. Choi, C. Morrison) of NASA-GRC Structures and Materials Adaptive Structures Technology Team are also acknowledged for discussions and suggestions.

\section{References}

1. Propulsion Directorate, AFRL/WPAFB (2000), High Cycle Fatigue (HCF) Program 1999 Annual Report, AFRL-PR-WPTR-2000-2004.

2. Kosmatka, J.: Experimental Spin Testing of Integrally Damped Composite Plates, NASA/CR-1998-207058, 1998.

3. Duffy, K. P., Bagley, R. L., and Mehmed, O.: On a Self-Tuning Impact Vibration Damper for Rotating Turbomachinery, AIAA-2000-3100, 2000.

4. Duffy, K., Brown, G., and Bagley, R.: Self-Tuning Impact Damper for Rotating Blades, U. S. Patent 6,827,551, December 2004.

5. Zhu, D., Miller, R. A., Duffy, K. P., and Ghosn, L. J.: High Temperature Damping Behavior of Plasma-Sprayed Thermal Barrier and Protective Coatings, ICACC-S2-039-2009, January 2009.

6. Duffy, K. P., Padula, S. A., II, and Scheiman, D. A.: Damping of High-Temperature Shape Memory Alloys, SPIE-6929-48, March, 2008.

7. J. B. Min, K. Duffy, B. B. Choi, C. R. Morrison, R. H. Jansen, and A. J. Provenza: A Resonant Damping Study Using Piezoelectric Materials, AIAA 2008-2335, 49 ${ }^{\text {th }}$ AIAA/ASME/ASCE/AHS/ASC Structures, Structural Dynamics, and Materials Conference, April 2008.

8. Choi, B., Morrison, C., and Min, J.: A Multi-Mode Blade Damping Control Using Shunted Piezoelectric Transducers with 
Active Feedback Structure, Propulsion-Safety and Affordable Readiness Conference, March 2009.

9. Kirsten P. Duffy, Andrew J. Provenza, Jeffrey J. Trudell, and James B. Min: Passively Shunted Piezoelectric Damping of Centrifugally-Loaded Plates, AIAA 2009-2524, 50th AIAA/ASME/ASCE/AHS/ASC Structures, Structural Dynamics, and Materials Conference, May 2009.

10. Lesieutre, G. A.: Vibration Damping and Control Using Shunted Piezoelectric Materials, The Shock and Vibration Digest, Vol. 30, No. 3, 1998, pp. 187-195.

11. Chopra, I.: Review of State of Art of Smart Structures and Integrated Systems, AIAA Journal, Vol. 40, No. 11, November 2002, pp. 2145-2187.

12. Hagood, N. W. and von Flotow, A.: Damping of Structural Vibrations with Piezoelectric Materials and Passive Electrical Networks, Journal of Sound and Vibration, Vol. 146, No. 2, 1991, pp. 243-268.

13. Hilbert, G. R., Pearson, D. D., and Crawley, E. F.: Method and Apparatus for Damping Vibration in Turbomachinery Components, U.S. Patent 6,299,410, 2001.

14. Livet, S., Collet, M., Berthillier, M., Jean, P., and Cote, J. M.: Turbomachinery Blades Damping Thanks to Optimized Shunted Piezoelectric Circuits, Proceedings of SPIE Smart Structures and Materials and Nondestructive Evaluation and Health Monitoring, SPIE-6928-12, March 2008.

15. Cross, C. J. and Fleeter, S.: Shunted Piezoelectrics for Passive Control of Turbomachine Blading Flow-Induced Vibrations, Smart Materials and Structures, Vol. 11, 2002, pp. 239-248.

16. Yu, H. and Wang, K. W.: Piezoelectric Networks for Vibration Suppression of Mistuned Bladed Disks, ASME Journal of Vibration and Acoustics, Vol. 129, No. 5, 2007, pp. 559-566.

17. P. Remington, D. Sutliff, and S. Sommerfeldt: Active Control of Low-Speed Fan Noise Using Actuators Mounted in Stator Vanes," AIAA 2003-3190, $9^{\text {th }}$ AIAA/CEAS Aeroacoustics Conference and Exhibit, May 2003, Hilton Head, SC.

18. ANSYS Multiphysics, ANSYS, Inc., Canonsburg, PA 15317.

19. Carnegie, W.: Vibrations of Rotating Cantilever Blading," J. of Mechanical Engineering Science, Vol. 1, No. 3 , 1959.

20. H. Allik, T. J. R. Hughes: Finite Element Method for Piezoelectric Vibration, Int. J. Numerical Methods in Eng., Vol. 2, 1970, pp. 151-157.

21. Ostergaard, D. F., Pawlak, T.P.: Three-Dimensional Finite Element for Analyzing Piezoelectric Structures, IEEE, Ultrasonics Symposium, Houston, pp. 639-644.

22.Humberto Ferreira Vinhais, Ricardo Cury Ibrahim, Emilio Carlos Nelli Silva: Simulation of a linear piezoelectric motor by using finite element method, ACBM symposium in mechatronics, Vol. 1, pp. 697-706, 2004.

23. J. Wang, D. Ostergaard: A finite element-electric circuit simulation method for piezoelectric transducer, Proceedings of IEEE utrasonics symposium, Vol. 2, 1999, pp. 1105-1108.

24. McCalla, M.C.: Fundamentals of computer-aided circuit simulation, Kluwer Academic, 1988.

25. Dimitris Varelis and Dimitris A. Saravanos: Small-Amplitude Free-Vibration Analysis of Piezoelectric Composite Plates Subject to Large Deflections and Initial Stresses, ASME Journal of Vibration and Acoustics, Vol. $128 \cdot$ No. $1 \bullet$ February 2006. 